Original paper

\title{
Antiviral therapy in hepatitis C-infected patients prevents relapse of diffuse large B cell lymphoma
}

\author{
Adriano Pellicelli', Valerio Giannelli', Valerio Zoli', Valeria Pellicelli², Anna Linda Zignego ${ }^{3}$ \\ 'Liver Unit, Department of Liver Transplant, Azienda Ospedaliera San Camillo Forlanini, Rome, Italy \\ "University of Rome "La Sapienza", Italy \\ ${ }^{3}$ MASVE Center University of Florence, Italy
}

\begin{abstract}
Introduction: Hepatitis C infection (HCV) is highly prevalent worldwide and has a well-known association with B-cell lymphoid malignancies. While several studies have demonstrated that antiviral therapy (AT) is effective to induce a complete hematological response in HCV-related low-grade B cell lymphoma, in HCV-related highgrade $B$ cell non-Hodgkin lymphomas such as diffuse large B cell lymphoma (DLBCL) chemotherapy is the only possible choice. However, the role of AT to reduce relapse of DLBCL after an effective chemotherapy containing rituximab (CT-R) has not been analyzed in previous studies. Therefore we analyzed whether patients with a sustained virological response (SVR) to AT had over time a reduction of lymphoma relapse compared to no-SVR patients.

Material and methods: The study included 21 consecutive HCV-infected patients affected by DLBCL. The patients were treated with AT (direct-acting antivirals or pegylated interferon alfa plus ribavirin) concomitantly or after CT-R. Over time, we evaluated relapse of DLBCL in patients treated with CT-R according to response to AT.

Results: An SVR was achieved in 16 of 21 patients. Five patients relapsed on AT with PegIFN/R (pegylated interferon plus ribavirin). Over time lymphoma relapse was more frequent in patients without a virological response compared with patients with an SVR ( $R R=12.0,95 \% \mathrm{Cl}: 1.66-86, p<0.01$ Fisher's exact test).

Conclusions: AT during or after CT-R is an important strategy to prevent relapse of DLBCL in HCV patients when the patients have achieved an SVR. Our results suggest that eradication of HCV infection may result in long-term prevention of B-cell non-Hodgkin's lymphoma relapse.
\end{abstract}

Key words: B cell lymphoma, HCV, chemotherapy, DAAs, relapse.

\section{Address for correspondence}

Adriano Pellicelli, Liver Unit, Department of Liver Transplant, Azienda Ospedaliera San Camillo Forlanini, Circonvallazione Gianicolense 87, 00152 Rome, Italy, phone: +393471196399, fax: +390658704667, e-mail: adriano.pellicelli@uniroma1.it

\section{Introduction}

Hepatitis C virus (HCV) is associated with a large spectrum on lymphoproliferative disorders. In nonHodgkin's lymphoma (NHL) prevalence of $\mathrm{HCV}$ infection is estimated to be about $15 \%$, which is approximately 8-10-fold higher than that reported in the general population $(1.5 \%)$ [1]. The histologic subtypes more closely related to $\mathrm{HCV}$ are represented by diffuse large B cell lymphoma (DLBCL), marginal zone lymphoma, and lymphoplasmacytic lymphoma [2]. The etiological link between HCV and DLBCL is probably due to the role of HCV in lymphomagenesis ("antigen-driven proliferation") [3]. It was demonstrated in several studies as, in low-grade HCV-related marginal zone lymphoma, eradication of HCV through antiviral therapy (AT) led to regression of lymphoma in the majority of cases [4]. Diffuse large cell lymphoma is a high-grade lymphoma and chemotherapy is the first form of treatment. The role of AT is less clearly defined. No sufficient data are available about the role of the new antiviral agents in DLBCL patients treated with chemotherapy containing rituximab (CT-R). The advent of directacting antivirals (DAAs) makes the treatment of $\mathrm{HCV}$ 
more appealing, especially in this difficult-to-treat population. The most recent updated hepatological (EASL) and hematological (NCCN) international guidelines suggest beginning the HCV treatment as the first therapeutic choice in the case of indolent lymphomas, but we lack a solid recommendation for DAAs in DLBCL due to the lack of data $[5,6]$.

Hence, we aimed to analyze the role of AT in preventing the recurrence of DLBCL in HCV-infected patients treated with CT-R. DAAs' efficacy and safety profile were also investigated.

\section{Material and methods}

The study cohort included 21 consecutive HCVpositive patients affected with DLBCL (12 males and 7 females) referred to our tertiary center of hepatology in Rome from January 2013 to December 2017, for a clinical and therapeutic evaluation for HCV treatment with a DAA or with Peg/RBV. The patients studied were under simultaneous observation for management of NHL disease at the hematology service of our hospital. All patients were treated with different chemotherapeutic regimens, all including rituximab. The patients were also treated with AT (DAAs or pegylated interferon plus ribavirin) concomitantly or after CT-R. All the patients were tested for liver fibrosis with a transient elastography measurement with a Echosens FibroScan device, model 502 (Echosens, Paris, France), or with liver biopsy (Ishak score) prior to the start of AT.

During antiviral treatment all the patients were followed up, with weekly evaluations of their liver function and monthly evaluation of HCV RNA. Adverse reactions to AT were recorded. In accordance with the aim of the study follow-up data were prospectively collected for up to five years after treatment conclusion, in order to define the disease-free-survival.

Sustained virological response (SVR) was defined as the disappearance of serum HCV RNA 12 or 24 weeks after the end of therapy according to type of AT.

Inclusion criteria were: diagnosis of aggressive DLBCL and indication for antineoplastic treatment (patients underwent the best treatment of choice, in accordance with the hematological guidelines in force at the time of diagnosis of neoplasia), together with genotype HCV active infection at the onset of the neoplastic disease. Exclusion criteria were: indolent or low-grade lymphoma, current and/or previous HBV infection, presence of clinical decompensated cirrhosis.

All patients gave their written informed consent to the data collection and the conduction of the study. The present study was conducted in compliance with the Declaration of Helsinki.
The NHL stage was defined by the Ann Arbor classification according to physical examination, total body computed tomography scan, bone marrow biopsy and biopsy of the organ involved. Complete hematological remission was defined as the absence of disease for at least one month after the end of chemotherapy. The statistical analysis was performed using IBM-SPSS (Statistical Package for Social Science) v.21 for McIntosh. Patients with SVR and without SVR were compared using the Mann-Whitney $U$ test. The cumulative risk for relapse of DLBCL during the follow-up was assessed through a Kaplan-Meier curve and the comparison between DLBCL patients with or without SVR was performed through the log-rank test.

\section{Results}

The patients' characteristics of our study population are reported in Table 1. Liver fibrosis was assessed prior to the start of treatment with AT by performing transient elastography or liver biopsy: 2 patients had mild liver fibrosis $(<7.0 \mathrm{kPa}), 13$ had liver stiffness that was compatible with moderate fibrosis $(7.0-9.5 \mathrm{kPa})$, 4 patients had severe fibrosis $(9.5-12.5 \mathrm{kPa})$. ChildPugh A liver cirrhosis $(>12.5 \mathrm{kPa})$ was revealed in 2 patients. Genotype 1 was the most represented genotype $(57.1 \%)$. All patients had a stage IV DLBCL. We evaluated relapse of DLBCL after CT-R in patients with or without an SVR to AT. An SVR was achieved in 16 of 21 patients. Five patients treated with PegIFN/ RBV combination therapy did not achieve an SVR and relapse of the neoplastic disease was noted in 4 patients. In our series, lymphoma relapse was more frequent in patients without a virological response compared to patients who achieved an SVR $(\mathrm{RR}=9.0$, 95\% CI: 1.27-63.00, $p<0.02$ Mann-Whitney $U$ test). No patients treated with DAAs during chemotherapy presented transaminase flare or liver function decompensation. All the characteristics of the patients are reported in Table 1.

The actuarial cumulative probability of being free from relapse of the neoplastic disease at 12, 24 and 48 months was $100 \%, 94 \%$ and $94 \%$ in SVR patients and $82 \%, 50 \%$ and $20 \%$ in no-SVR patients, respectively (Figure 1).

\section{Discussion}

While different studies have shown a benefit of AT in HCV-related low-grade lymphoma in terms of lymphoma remission, only a few studies have demonstrated in HCV-positive DLBCL efficacy of AT always combined with CT-R in order to reinforce the remission 
Table 1. Patients with diffuse large B cell lymphoma treated with different antiviral treatments

\begin{tabular}{|c|c|c|c|c|c|c|c|c|c|c|}
\hline Pt & $\begin{array}{c}\text { Age } \\
\text { (years) }\end{array}$ & $\begin{array}{l}\mathrm{NHL} \\
\text { stage }\end{array}$ & $\begin{array}{c}\text { HCV } \\
\text { genotype }\end{array}$ & $\begin{array}{c}\text { Fibrosis stage } \\
\text { (METAVIR) }\end{array}$ & CT & $\begin{array}{l}\text { HR after } \\
\text { CT-R }\end{array}$ & AT & Time AT & SVR & $\begin{array}{c}\text { Hem. } \\
\text { Rel/FU (m) }\end{array}$ \\
\hline 1 & $23 / M$ & 3 & $1 b$ & F2 & $\mathrm{R}-\mathrm{CHOP}$ & $C R$ & Peg/RBV & Post & Y & No/48 \\
\hline 2 & $44 / F$ & 4 & 2 & F2 & $\mathrm{R}-\mathrm{CHOP}$ & $C R$ & $\mathrm{Peg} / \mathrm{RBV}$ & Post & Y & No/52 \\
\hline 3 & $68 / M$ & 4 & 2 & F2 & $\mathrm{R}-\mathrm{CHOP}$ & $C R$ & Peg/RBV & Post & No & Yes/48 \\
\hline 4 & $71 / M$ & 4 & 2 & F3 & R-VCP & $C R$ & Peg/RBV & Post & No & Yes/18 \\
\hline 5 & $50 / \mathrm{M}$ & 4 & $1 b$ & $\mathrm{~F} 1$ & $\mathrm{R}-\mathrm{CHOP}$ & $C R$ & Peg/RBV & Post & No & Yes/43 \\
\hline 6 & $74 / F$ & 4 & $1 b$ & F3 & $\mathrm{R}-\mathrm{CHOP}$ & $C R$ & Peg/RBV & Post & Y & Yes/20 \\
\hline 7 & $58 / \mathrm{M}$ & 4 & $1 \mathrm{a}$ & F2 & $\mathrm{R}-\mathrm{CHOP}$ & $C R$ & Peg/RBV & Post & Y & No/58 \\
\hline 8 & $45 / M$ & 4 & $1 b$ & $\mathrm{~F} 1$ & $\mathrm{R}-\mathrm{CHOP}$ & $C R$ & $\mathrm{Peg} / \mathrm{RBV}$ & Post & No & Yes/18 \\
\hline 9 & $72 / \mathrm{M}$ & 4 & 2 & F2 & R-VCP & $C R$ & Peg/RBV & Post & No & No/57 \\
\hline 10 & $64 / M$ & 4 & $1 b$ & F3 & $\mathrm{R}-\mathrm{CHOP}$ & $C R$ & Peg/RBV & Post & Y & No/69 \\
\hline 11 & $59 / \mathrm{F}$ & 4 & $1 \mathrm{a}$ & F2 & $\mathrm{R}-\mathrm{CHOP}$ & $C R$ & $\mathrm{Peg} / \mathrm{RBV}$ & Post & Y & No/46 \\
\hline 12 & $40 / F$ & 4 & $1 b$ & F2 & R-VCP & $C R$ & SOF/SMV & Post & Y & No/26 \\
\hline 13 & $54 / \mathrm{M}$ & 4 & 3 & F2 & $\mathrm{R}-\mathrm{CHOP}$ & $C R$ & SOF/DCV & During & Y & No/20 \\
\hline 14 & $55 / \mathrm{M}$ & 4 & $1 \mathrm{a}$ & F4 & $\mathrm{R}-\mathrm{CHOP}$ & $C R$ & SOF/SMV & Post & Y & No/22 \\
\hline 15 & $74 / F$ & 4 & 2 & F2 & $\mathrm{R}-\mathrm{CHOP}$ & $C R$ & SOF/RBV & During & Y & No/23 \\
\hline 16 & $55 / \mathrm{M}$ & 4 & $1 a$ & F3 & $\mathrm{R}-\mathrm{CHOP}$ & $C R$ & SOF/SMV & Post & Y & No/28 \\
\hline 17 & $40 / \mathrm{M}$ & 4 & 3 & F2 & $\mathrm{R}-\mathrm{CHOP}$ & $C R$ & SOF/LDV & Post & Y & No/20 \\
\hline 18 & $67 / F$ & 4 & $1 b$ & F2 & $\mathrm{R}-\mathrm{CHOP}$ & $C R$ & Omb-Par/R-Das & Post & Y & No/22 \\
\hline 19 & $52 / \mathrm{F}$ & 4 & 3 & $\mathrm{~F} 2$ & R-BEND & $C R$ & SOF/DCV & During & Y & No/22 \\
\hline 20 & $59 / \mathrm{M}$ & 4 & 4 & F4 & $\mathrm{R}-\mathrm{CHOP}$ & $C R$ & SOF/DCV/RBV & During & Y & No/32 \\
\hline 21 & $66 / F$ & 4 & $1 b$ & F2 & $\mathrm{R}-\mathrm{CHOP}$ & $C R$ & SOF/SMV & During & Y & No/28 \\
\hline
\end{tabular}

$P t$ - patient, AT - antiviral treatment, HR after CT-R - hematological response after chemotherapy containing rituximab, $C R$ - complete hematological response, $R$-CHOP - rituximab plus cyclophosphamide, vincristine, doxorubicin, vincristine, prednisolone, R-VCP - rituximab plus cyclophosphamide, vincristine, prednisolone, Peg - pegylated interferon, RBV - ribavirin, Post - post-chemotherapy, During - during chemotherapy, SVR - sustained virological response, SOF - sofosbuvir, SMV - simeprevir, DCV - daclatasvir, LDV - ledipasvir, FU - follow-up from the end of AT, Omb - ombitasvir, Par - paritaprevir, R-Das - ritonavir/dasabuvir, BEND - bendamustine

and to reduce relapse of neoplastic disease. Persico et al. demonstrated that in $20 \mathrm{HCV}$-positive patients undergoing CT-R for DLBCL and treated with DAAs there was better overall survival and disease-free survival in 52 weeks of follow-up compared to a control group of HCV-positive patients treated only with CT [7]. However, in this study $45 \%$ of the control group was treated with a CT regimen without rituximab, which could have influenced the results. Several studies have shown that disease-free survival (DFS) and overall survival (OS) is lower in patients with DLBCL treated with CT not containing rituximab compared to patients treated with CT-R (20\% vs. 36.5$)[8,9]$. For this reason it is difficult in this study to extrapolate the conclusion about DFS and OS due to the heterogeneous group of patients. Our brief report has the strength of confirming in a small series of patients the effective role of SVR after AT for the long-term remission of neoplastic disease, especially in those patients treated with DAAs.

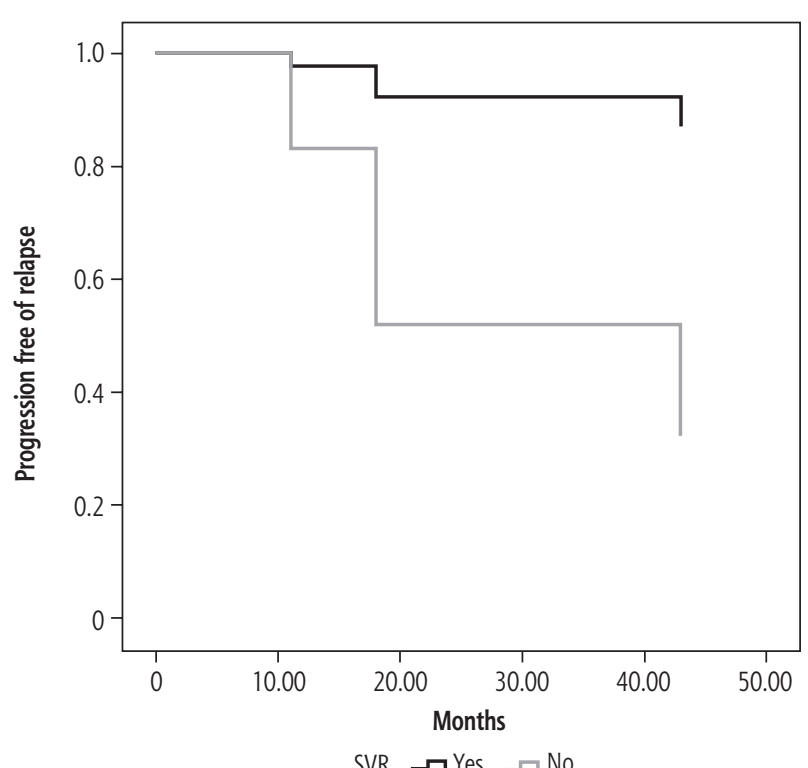

Fig. 1. Comparison of 50 months relapse of diffuse large B cell lymphoma in HCV-infected patients with or without sustained virological response (SVR) 
Hosryetal. demonstrated that $\mathrm{HCV}$-positive patients never treated with AT had DLBCL more refractory to first-line chemotherapy than that in HCV-negative patients $(33 \%$ vs. $17 \% ; p=0.05)$ and had more progressive lymphoma, though not statistically significantly, compared to HCV-negative patients ( $50 \%$ vs. $32 \%$; $p=0.09$ ) or patients given AT ( $50 \%$ vs. $27 \%$; $p=0.06$ ). This result could suggest that DLBCL in HCV-infected patients is more refractory to CT-R compared to HCV-negative patients. Moreover, they found that patients never given AT had worse 5-year OS rates than did treated patients ( $\mathrm{HR}=2.3,95 \% \mathrm{CI}: 1.01-5.30, p=0.04)$. In this study there was not found a reduction of lymphoma relapse after CT-R between HCV-treated and non-treated patients ( $41 \%$ vs. $46 \%, p=0.7$ ) [10]. We have some concern about this result because the authors did not analyze the lymphoma relapse rate between HCV-treated patients who achieved a SVR and those who did not. In our opinion the real impact of AT on lymphoma relapse is difficult to extrapolate in this study. Michot et al. observed a positive association between AT and OS in DLBCL patients. They stated that AT reinforced the results of a successful CT-R [11]. It is our opinion that AT during or after chemotherapy is an important strategy to prevent relapse of DLBCL in HCV-infected patients when the patients achieved a SVR. This hypothesis may be based in part on sustained B cell activation and inhibition of B cell apoptosis by HCV [12]. Eradication of hepatitis $C$ can turn off the persistent activation of B lymphocytes by HCV. Another important consideration must be done. Furthermore, we have to consider that the use of chemotherapy in combination with rituximab for the treatment of DLBCL in patients infected with $\mathrm{HCV}$ can produce different degrees of liver dysfunction [13]. A rare case of cholestatic hepatitis $C$ was also published; it occurred in a patient with DLBCL and HCV infection [14]. DAAs demonstrated excellent safety and seem not to have pharmacological interactions with CT-R. Several reports have described cases of DLBCL treated at the same time with CT-R and DAAs without adverse events and a reduction of liver toxicity $[15,16]$. Cicero in the trial defending Sextus Roscius, who was accused of patricide, asked "Cui bono?" (Who benefits?). This same question needs to be answered in HCV-infected patients with DLBCL in the era of DAAs. We believe the answer lies both in the prevention of relapse of DLBCL and reduction of liver damage during CT-R, but also in the prevention of HCV-related complications after cure of DLBCL.

In conclusion, our study confirms that in HCVassociated DLBCL, AT reduces the lymphoma relapse rate when an SVR is achieved. Furthermore, a DAA combined with CHOP-R is safe and seems to reduce the risk of hepatitis $\mathrm{C}$ flare and hepatic toxicity due to immunochemotherapy.

\section{Disclosure}

The authors report no conflict of interest.

\section{References}

1. Dal Maso L, Franceschi S. Hepatitis C virus and risk of lymphoma and other lymphoid neoplasms: a meta-analysis of epidemiologic studies. Cancer Epidemiol Biomarkers Prev 2006; 15: 2078-2085.

2. De Sanjose S, Benavente Y, Vladic CM. Hepatitis C and non-Hodgkin lymphoma amon 4784 cases and 6269 controls form the International Lymphoma Epidemiology Conortium. Clin Gastroenterol Hepatol 2008; 6: 451-458.

3. Peveling-Oberhag J, Arcaini L, Hansmann ML, Zeuzem S. Hepatitis C-associated B-cell non-Hodgkin lymphomas. Epidemiology, molecular signature and clinical management. J Hepatol 2013; 59: 169-177.

4. Merli M, Carli G, Arcaini L, Visco C. Antiviral therapy of hepatitis $\mathrm{C}$ as curative treatment of indolent B-cell lymphoma. World J Gastroenterol 2016; 22: 8447-8458.

5. National Comprehensive Cancer Network (NCCN) Guidelines. Version 4.2014. Non Hodgkin's Lymphoma.

6. EASL Recommendations on treatment of hepatitis C 2016. J Hepatol 2017; 66: 153-194.

7. Persico M, Aglitti A, Caruso R, et al. Efficacy and safety of new direct antiviral agents in hepatitis $\mathrm{C}$ virus-infected patients with diffuse large B-cell non-Hodgkin's lymphoma. Hepatology 2018; 67: 48-55.

8. Coiffier B, Pfreundschuh M, Stahel R, et al. Aggressive lymphoma: improving treatment outcome with rituximab. Anticancer Drugs 2002; 13 Suppl 2: S43-50.

9. Coiffier B, Thieblemont C, Van Den Neste E, et al. Long-term outcome of patients in the LNH-98.5 trial, the first randomized study comparing rituximab-CHOP to standard $\mathrm{CHOP}$ chemotherapy in DLBCL patients: a study by the Groupe d'Etudes des Lymphomes de l'Adulte. Blood 2010; 116: 2040-2045.

10. Hosry J, Mahale P, Turtutto F, et al. Antiviral therapy improves overall survival in hepatitis $\mathrm{C}$ virus infected patients who develop diffuse large B-cell lymphoma. Int J Cancer 2016; 139: 2519-2528.

11. Michot JM, Canioni D, Driss H, et al. Antiviral therapy is associated with a better survival in patients with hepatitis $\mathrm{C}$ virus and B-cell non Hodgkin lymphomas, ANRS HC-13 lympho-C study. Am J Hematol 2015; 90: 197-203.

12. Zignego AL, Giannini C, Gragnani L. HCV and lymphoproliferation: Clin Dev Immunol 2012; 2012: 980942.

13. Zaky AH, Bakry R, El-sayed MI, et al. Impact of treatmentrelated toxicity on outcome of HCV-positive diffuse large B-cell lymphoma in rituximab era. Hematology 2014; 19: 412-416.

14. Pellicelli AM, D’Ambrosio C, Dessanti ML, et al. Cholestatic hepatitis $\mathrm{C}$ after chemotherapy containing rituximab in diffuse large B cell lymphoma. Ann Hepatol 2015; 14: 756-761.

15. Alric L, Besson C, Lapidus N, et al. Antiviral treatment of HCV-infected patients with B-cell non-Hodgkin lymphoma: ANRS HC-13 lympho-C study. PLoS One 2016; 11: e0162965.

16. Ewers EC, Shah PA, Carmichael MG, Ferguson TM. Concurrent systemic chemoimmunotherapy and sofosbuvir based antiviral treatment in a hepatitis $\mathrm{C}$ virus infected patient with diffuse large B cell lymphoma. Open Forum Infect Dis 2016; 3: ofw223. 